\title{
Family caregivers' assessment of symptoms in persons with dementia using the GBS- scale: differences in rating after psychosocial intervention - an 18-month follow-up study
}

\author{
This article was published in the following Dove Press journal: \\ Clinical Interventions in Aging \\ 17 December 2010 \\ Number of times this article has been viewed
}

\author{
Beth Dahlrup \\ Eva Nordell \\ Signe Andrén \\ Sölve Elmståhl \\ Department of Health Sciences, \\ Division of Geriatric Medicine, \\ Lund University, Sweden
}

Correspondence: Beth Dahlrup Department of Health Sciences, Division of Geriatric Medicine, CRC, Ent 72, Build 28, FI.I3, Skane University Hospital SE-205 02 Malmo, Sweden $\mathrm{Tel}+4640391314$

$\mathrm{Fax}+46391313$

Email beth.dahlrup@med.lu.se

\begin{abstract}
The purpose of this study was to examine if psychosocial intervention for family caregivers made any differences in describing symptoms of dementia in the persons they cared for. The study population comprised family caregivers of persons aged 70 years and older receiving social services and diagnosed with dementia disorders. A group of 129 family caregivers underwent psychosocial intervention including education, information, and provision of a support group, while 133 family caregivers did not and these formed the control group. Family caregivers were followed-up every 6 months for a total of 18 months. They rated intellectual, emotional, and activity of daily living (ADL) functions in persons with dementia using the Gottfries-Bråne-Steen scale (GBS-scale). Family caregivers who underwent psychosocial intervention rated the intellectual and emotional symptoms of dementia significantly higher 6 months later compared to controls and the effect was sustained during the 18-month follow-up irrespective of relationship and education. Most notably, decrease in function of recent memory, ability to increase tempo, long-windedness, distractibility, and blunting were better identified. Our findings suggest that the family caregivers who underwent psychosocial intervention achieved better understanding of different symptoms and the behaviors of dementia. These findings may explain earlier findings of positive effects after psychosocial intervention on family caregivers' sense of burden, satisfaction, and ability to delay nursing home placement.
\end{abstract}

Keywords: intervention, dementia, family caregivers, education, GBS-scale

\section{Introduction}

In clinical practice, the family caregiver of the person with dementia often serves as the main source of information regarding cognitive and functional decline. ${ }^{1}$ Proxy information is considered very valuable since the persons with the disease may have difficulty assessing their own abilities and, as a result, tend to both over- and underestimate their shortcomings. The family caregiver's assessment generally correlates with the patient's cognitive decline and is considered more useful than his or her own assessment. ${ }^{2,3}$ Onor et al also identified major differences between how persons in early stages of dementia and their family caregivers perceived cognitive and behavioral disorders. ${ }^{3}$ The persons with dementia underestimated deficits that the family caregivers considered to be serious and disabling. Loewenstein et al compared the assessment of the family caregivers to objective measures. ${ }^{4}$ The result indicated that the family caregivers were accurate in describing functional deficits in the persons with mild symptoms of dementia, but 
overestimated the ability of more impaired persons to tell time, identify and count currency, and to manage eating utensils. They found that adult children were less accurate in their assessment than spouses, presumably because the adult children usually had less opportunity to observe daily behaviors. Lowenstein et al also suggested that assessment might be influenced by denial of illness by the family caregivers as well as the possibility that the caregivers were burdened and depressed. ${ }^{4}$ In a study assessing quality of life (QoL), persons with Alzheimer's disease had a more positive view regarding their QoL than had professionals. Spouses had a more positive perception of the persons' QoL than the adult children. ${ }^{5}$ These reported findings raised questions whether psychosocial interventions for family caregivers of persons with dementia could also influence the caregivers' assessment of the symptoms of dementia. To our knowledge, this has not been reported previously. Also, longitudinal studies regarding family caregivers' assessment of symptoms are sparse. Previous investigations, including the participants studied here, have demonstrated that psychosocial intervention including information, education, and conversation groups showed significant and positive effects on the family caregivers' burden and satisfaction. ${ }^{6,7}$ Psychosocial intervention for adult children caregivers also delayed nursing home placement by approximately 6 months. ${ }^{8}$ There is a need to evaluate whether these reported effects could be a result of better understanding about the symptoms of dementia after psychosocial intervention. The purpose of this study was to examine if psychosocial intervention for family caregivers made any difference in describing symptoms of dementia in the persons they cared for.

\section{Methods}

\section{Participants}

In order to find family caregivers to persons with dementia, a letter of invitation was sent to persons $>70$ years of age receiving social services in 2 out of 10 districts from one and the same municipality in southern Sweden. The districts were chosen to ensure similar sociodemographic structures and similar levels of public service. The two districts had similar gender proportions, ie, $72 \%$ versus $68 \%$ women. The proportions of married persons were $29 \%$ versus $32 \%$ and widowed $51 \%$ versus $54 \%$. Employed staff in elderly care was 105 versus 106 per 1000 inhabitants $>80$ years and day care services 5.3 versus 4.4 per 1000 inhabitants $>80$ years. ${ }^{6-8}$

The invitation letter was sent to 2721 people who were invited to participate in a study which included an assessment of their general health and cognition. A member of the family was also invited to participate.

A second letter was later sent to those who had not responded. Home visits for the persons who responded ( $n=1656)$ were carried out by a registered nurse $(\mathrm{RN})$ who assessed cognition according to the Mini-Mental State Examination (MMSE). ${ }^{9}$ If symptoms of cognitive decline were found and/or the MMSE score was $<24$, the person was invited to undergo a medical examination. In total, 571 individuals agreed and underwent clinical examination by a physician. They were also interviewed by a registered nurse and physician in order to establish a diagnosis of dementia. On this occasion, the family caregivers were separately interviewed by the same professionals in order to enhance the examination. A dementia diagnosis, according to the Diagnostic and Statistical Manual of Mental Disorders IV (DSM-IV) ${ }^{10}$ criteria, was established in 415 persons who formed dyads, ie, the family caregiver and the person with dementia. ${ }^{6-8}$ Of these, 262 dyads were available for this study. Those with dementia who had moved to a nursing home or had died, who had family caregivers who were not a spouse or child, family caregivers who declined participation, and/or those with an incomplete questionnaire were excluded from the study $(n=153)$. Family caregivers and the persons with dementia from one of the districts comprised the dyads in the intervention group $(n=129)$ and the control group was comprised of the family caregivers and persons with dementia from the other district $(n=133)$.

Written consent was obtained from all participants.

The study design was quasi-experimental and a part of a recently completed longitudinal cohort study entitled the Malmo Intervention study of family caregivers to persons with Dementia (MIND). ${ }^{6-8}$

\section{Procedures}

Baseline data were collected between August 1999 and April 2003 for the intervention group and from January 2000 to May 2005 for the control group. ${ }^{7}$ The participation of the family caregiver continued until the person with dementia moved to a nursing home or died or until five years of living in their own homes had passed. ${ }^{8}$ For this particular study, the participants were followed for 18 months.

With the assistance of the family caregiver, a RN assessed baseline symptoms of dementia using the GottfriesBråne-Steen scale (GBS-scale), ${ }^{11}$ the severity of dementia using the Berger scale, ${ }^{12}$ and functional disabilities with the Katz Index of activities of daily living (ADL). ${ }^{13}$ These instruments were used throughout the study. 
A questionnaire was sent by mail at baseline and every 6 months to the family caregivers regarding sociodemographic data, type of care provided by the municipality and/or the family caregivers, and if the need for assistance was met. The family caregivers also registered caregiver burden, perceived health, sense of coherence, and satisfaction in the role as a family caregiver. These data have been presented elsewhere. ${ }^{6}$ Data were collected in the postal questionnaire regarding the GBS-scale. Data concerning the Berger scale and Katz index were collected by a $\mathrm{RN}$ through a telephone interview in connection with the postal questionnaire. As a complement to the questionnaires, telephone interviews with all family caregivers were also carried out between the postal questionnaires to update changes in the care provided by the municipality and/ or the caregivers. In addition, the family caregivers had the opportunity to attend ad hoc counseling sessions regardless of being in the intervention or the control group. ${ }^{8}$

\section{Instruments}

\section{Mini-Mental State Examination (MMSE)}

The MMSE$^{9}$ is a widely used instrument to define cognitive impairment and consists of a brief examination including 11 questions. The questionnaire tests a person's orientation, attention, calculation, immediate and shortterm recall, language, and motor skills. The maximum score is 30 . A score $<24$ is considered to show cognitive impairment. ${ }^{9,14}$

\section{The Gottfries-Bråne-Steen scale (GBS-scale)}

The GBS-scale ${ }^{11}$ was constructed for rating dementia syndrome and the changes of symptoms over time and was first published in 1982. The GBS-scale is based on a semistructured interview and observation. The GBS-scale is comprised of three subscales measuring intellectual, emotional, and motor functions, as well as a section measuring symptoms characteristic of dementia syndromes. The intellectual functions are measured by 12 items (GBS-I): orientation to person, time, and space; recent and distant memory; wakefulness; concentration; ability to increase tempo; absentmindedness; long-windedness; distractibility; and language disturbances. The emotional subscale (GBS-E) has three items: emotional blunting, emotional lability, and motivation. The ADL functions (GBS-ADL) consists of six items: dressing, food intake, physical and spontaneous activities, personal hygiene, and ability to control bladder and bowel. Each item is scored on a 7-point scale where 0 is equivalent to normal function and 6 means severe symptoms. By adding the scores from the items in each subscale, an overview of the degree of dementia is obtained representing a range from an absence of symptoms to mild, moderate, or severe symptoms of dementia. The GBS-scale can be used to study the changes in symptoms in persons with dementia over time and also to view the effects of different interventions. The GBS-scale was found to be valid and highly reliable. ${ }^{15}$

\section{The Berger scale}

The Berger scale ${ }^{12}$ was chosen to rate the severity of dementia by describing social dependency. The construction of the Berger scale is hierarchical and follows the patterns of progression in dementia disorders. The scale consists of 6 classes where class 1 represents a person who, although forgetful, is still able to function in any surroundings. Class 2 describes a person able to function only in familiar surroundings. In class 3 , the person needs direction to function and responds appropriately to instruction. Class 4 represents a person in need of assistance to function and class 5 describes a person still ambulatory, but who cannot fully communicate. Finally, class 6 represents a person who is bedridden or confined to a chair and unable to communicate appropriately. The scale has previously been used in studies focusing on the progression of dementia diseases. ${ }^{7,16,17} \mathrm{In}$ this study, the scores were dichotomized into low (classes 1-2) and high severity of dementia (classes 3-6) in accordance to the work of Andrén and Elmståhl. ${ }^{7}$

\section{The Katz index of ADL}

The Katz index ${ }^{13}$ assesses a person's function in ADL, in the areas of bathing, dressing, toileting, transferring, continence, and food intake. The index is based on the premise that the loss of function in a person's ADL presents itself in a given order: A indicates independence in performing every activity; $\mathrm{B}$ shows independence in all but one activity; $\mathrm{C}$ represents independence in all but two activities and so forth. $G$ indicates dependency in all activities The internal consistency has been shown to be high, interrater variability low, and validity good. ${ }^{18}$ In this study, the scores were dichotomized into low (classes A-B) and high social dependency (classes C-G) according to Andrén and Elmståhl. ${ }^{7}$

\section{Intervention}

Intervention started 2-6 weeks after the person with dementia was diagnosed. Each group comprised approximately eight family caregivers, consisting of both spouses and adult children. The program included general education about the most common dementia disorders, consequences and prognosis of the disease, behavioral symptoms and ways of responding to problematic behavior, as well as information about available services in 
the community, legislation, and costs. The educational section was followed by group discussions. The program was led by a RN and a counselor and the groups met 2 hours weekly for 5 weeks. In addition to the program, the family caregivers were offered the opportunity to continue with conversation groups under the supervision of the counselor. Almost half of the caregivers chose this opportunity. The group met twice every month for 90 minutes for 3 months. ${ }^{7}$

\section{Data analyses}

Analyses were carried out with SPSS $^{\circledR} 17.0$ (IBM, Somers, NY). Two-tailed independent-sample $t$-tests were used for normally distributed outcome variables. Chi-square tests were used to compare dichotomous variables. A $P$ value $\leq 0.05$ was considered significant

The GBS-scale with its individual items was also analyzed at 18 months in the subgroup with dyads where the person with dementia still lived at home $(n=144)$.

\section{Ethical aspects}

Ethical approval was obtained prior to the data collection from the Ethical Committee of Lund University, Sweden (LU-1997-573).

\section{Results}

\section{Characteristics of family caregivers}

The mean age of the family caregivers was 61 years in the intervention group and 62 years among the controls and the proportion of those who were spouses was $30 \%$ and $24 \%$ respectively. The level of education differed between the groups with a higher percentage $(57 \%)$ of university and college education among the caregivers in the intervention group versus $43 \%$ in the controls (Table 1).

\section{Characteristics of persons with dementia}

The mean score for MMSE was 19 for the persons with dementia in the intervention group and 18 amongst the controls. In both groups, the individuals suffering from dementia had a similar age and gender distribution (Table 1).

The mean score of the GBS-I subscale was 16.8 in the intervention group and 22.8 among the controls $(P<0.001)$ and corresponding values for the GBS-E subscale were 2.4 and 4.4 respectively $(P<0.001)$. The mean score of the GBS-ADL subscale was 5.8 in the intervention group and 7.2 among the controls $(P=0.07)$. For both groups, the mean score of the intellectual, emotional, and ADL-scales represents mild symptoms of dementia. Low severity of dementia, according to the Berger scale, was found in $>70 \%$

Table I Demographic characteristics at baseline $(n=262)$

\begin{tabular}{|c|c|c|c|}
\hline & $\begin{array}{l}\text { Intervention group } \\
n=129\end{array}$ & $\begin{array}{l}\text { Control group } \\
n=133\end{array}$ & $P$ value \\
\hline \multicolumn{4}{|l|}{ Family caregivers } \\
\hline Mean age, years (SD) & $61(12.9)$ & $62(12.6)$ & 0.94 \\
\hline Women \% & 58 & 65 & 0.28 \\
\hline \multicolumn{4}{|l|}{ Educational level } \\
\hline Upper secondary school or less \% & 44 & 56 & \\
\hline University and university colleges \% & 57 & 43 & 0.05 \\
\hline \multicolumn{4}{|l|}{ Relationship } \\
\hline Spouses \% & 30 & 24 & \\
\hline Adult children \% & 70 & 76 & 0.32 \\
\hline \multicolumn{4}{|l|}{ Persons with dementia } \\
\hline Mean age, years (SD) & $84(5.7)$ & $85(5.9)$ & 0.06 \\
\hline Women \% & 77 & 74 & 0.57 \\
\hline Living alone \% & 68 & 74 & 0.31 \\
\hline MMSE mean score (SD) & $19(5.2)$ & $18(5.0)$ & 0.12 \\
\hline \multicolumn{4}{|l|}{ GBS subscales, mean score (SD) } \\
\hline Intellectual & $16.8(9.3)$ & $22.8(10.7)$ & $<0.001$ \\
\hline Emotional & $2.4(2.0)$ & $4.4(3.4)$ & $<0.001$ \\
\hline$A D L$ & $5.8(6.1)$ & $7.2(6.3)$ & 0.07 \\
\hline \multicolumn{4}{|l|}{ Berger scale } \\
\hline Low severity of dementia \% & 72 & 46 & \\
\hline High severity of dementia \% & 28 & 54 & $<0.001$ \\
\hline \multicolumn{4}{|l|}{ Katz index of ADL } \\
\hline Low dependency \% & 77 & 71 & \\
\hline High dependency \% & 23 & 29 & 0.33 \\
\hline
\end{tabular}

Abbreviations: SD, standard deviation; MMSE, mini-mental state examination; GBS, Gottfries-Bråne-Steen; ADL, activity of daily living. 
of the intervention group compared to $<50 \%$ in the controls $(P<0.001)$. The majority in both groups showed low dependency according to the Katz index of ADL (Table 1).

\section{The GBS-scale}

At 6 months, the score in the GBS-I subscale was rated higher by the family caregivers in the intervention group compared to the controls with mean differences of 12.7 and 5.3 respectively $(P<0.001)$ (Table 2$)$, giving a mean score of 28.9 for the persons with dementia in the intervention group and 27.9 in the controls $(P=0.56)$. These scores correspond to moderate symptoms of dementia on the intellectual subscale. Also, the ratings of the GBS-E and GBS-ADL were higher in the intervention group compared to controls at 6 months with mean differences of 2.9 and 0.9 $(P<0.001)$ for the GBS-E and 4.2 and $2.1(P=0.01)$ for the GBS-ADL (Table 2). The mean score in the GBS-E subscale for the persons with dementia in the intervention group was 5.2 versus 5.1 in the controls $(P=0.88)$. The mean scores for the GBS-ADL subscale were 9.8 and 9.0 respectively $(P=0.41)$.

At 12 months, the family caregivers in both groups continued to rate an increase of symptoms. Family caregivers in the intervention group rated the symptoms significantly higher than the family caregivers in the control group with regards to GBS-I $(P=0.02)$ and the GBS-E subscales $(P=0.04)$ (Table 2$)$. There were no significant differences in the GBS-ADL subscale ratings between the groups $(P=0.53)$. The mean score of the GBS-I for the persons with dementia in the intervention group was 29.9 versus 31.4 in the control group $(P=0.49)$, the GBS-E was 5.9 and $6.4(P=0.50)$, and the GBS-ADL subscale was 9.8 versus $11.6(P=0.07)$.

The same trend was seen at 18 months (Table 2). The family caregivers in the intervention group rated a greater increase of symptoms in all subscales, although these were not significant compared to controls.

\section{Assessment of the survivors}

When analyses were done of the family caregivers' ratings for the 18-month survivors in the intervention $(\mathrm{n}=76)$ and control group $(n=68)$, similar results were found in the three subscales for the survivors as for the entire group $(n=262)$. At baseline, the persons with dementia in the control group were rated with significantly higher degrees of symptoms in the GBS-I subscale. The mean score of GBS-I differed by 6.9 points $(P<0.001)$. When looking at the individual items, orientation in space, recent memory, wakefulness, ability to increase tempo, absentmindedness, long-windedness, and

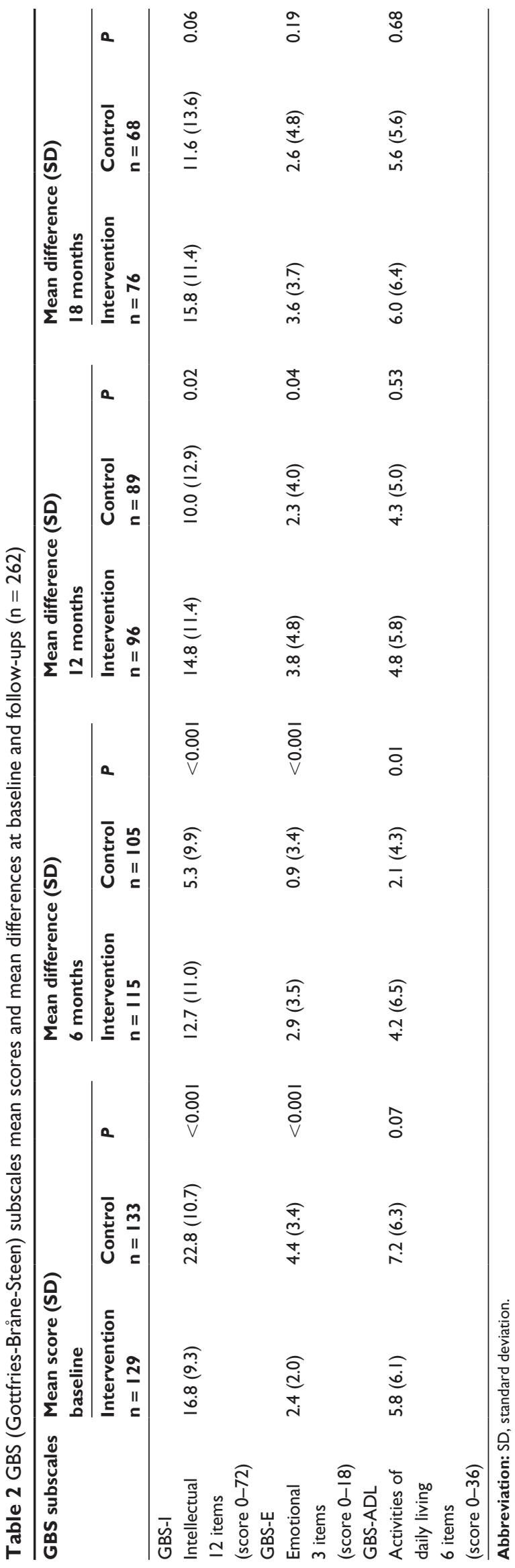


distractibility, each showed significant differences (Table 3). The GBS-E subscale differed by 1.6 points $(P<0.001)$ at baseline and in the individual items significant differences were demonstrated in two out of three items: emotional blunting and lability. The GBS-ADL subscale differed by 1.1 $(P=0.22)$ and significance was noted in two individual items: physical activities and continence (Table 3 ).

At 6 months, the mean differences were significant in both the GBS-I $(P=0.002)$ and GBS-E subscales $(P=0.009)$, indicating that the family caregivers in the intervention group were observing more symptoms (Figure 1). This was also evident in the individual items (Table 3). The GBS-ADL subscale and its individual items showed no significant differences.

The same pattern was demonstrated at 12 and 18 months and significant mean differences were seen mainly in the same individual items in the GBS-I and GBS-E subscales during the total follow-up (Table 3 and Figure 1). The family caregivers in the intervention group rated a higher number of symptoms in relation to lack of orientation to space, recent memory, ability to increase tempo, absentmindedness, long-windedness, distractibility, and emotional blunting up to 18 months later as compared to the controls (Table 3). No differences in assessment were noted for the GBS-ADL subscale between groups during follow-up (Figure 1).

Ratings of the three subscales and their individual items were analyzed for spouses and adult children in the intervention group and the control respectively. No differences were found between spouses and adult children within the groups, rather, the differences appeared only between the invention group and the controls. The difference in educational level between the family caregivers in the intervention group and controls did not affect the results. Dividing family caregivers that participated in the conversation group following the intervention to those who did not join this group, revealed no distinction in the ratings.

\section{The Berger scale}

At 6 months, assessments of the individuals with dementia in both the intervention and control group revealed a progression of the disease according to the Berger scale. Both groups were assessed and indicated a high severity of dementia (score 3-6 by the Berger scale) in 75\% of the intervention group and $73 \%$ among the controls $(P=0.72)$. The corresponding value at 12 months was $88 \%$ versus $77 \%$ $(P=0.05)$ and at 18 months $90 \%$ versus $83 \%$ respectively.

\section{The Katz index of ADL}

The assessment at 6 months revealed that $59 \%$ of the persons with dementia in the intervention group and $49 \%$ among the controls were rated with high dependency according to the Katz index of ADL $(P=0.16)$. This pattern was sustained at 12 months, when $71 \%$ of the persons with dementia in the intervention group versus $75 \%$ among the controls were rated with high dependency $(P=0.53)$. At 18 months, $70 \%$ in both groups were rated highly dependent in performing ADL $(P=0.99)$.

\section{Discussion}

The results in this study demonstrate that family caregivers who underwent psychosocial intervention rated the changes of symptoms higher between the follow-ups on the GBS-scale. This was more predominant in symptoms related to intellectual and emotional functions. Although not significant in all follow-ups, the trend was present at all occasions. We expected, due to the progression of dementia disorders, that family caregivers in both groups would assess deterioration during the study period, which we found to be the case. However, the assessment by the family caregivers in the intervention group was still higher. Thus, we assume that better understanding about the disease and its symptoms made the family caregivers in the intervention group more aware of the different behaviors occurring and, further, that these behaviors could be caused by the dementia disorder. This replicates earlier findings by Pinquart and Sörenson. ${ }^{19}$ In their review of the effectiveness of 76 different interventions for caregivers to persons with dementia, the authors established that psychoeducational intervention led to an improvement in caregivers' ability to cope and a better knowledge of the disease. ${ }^{19}$ A metaanalysis of 34 interventions showed that the family caregivers to persons with dementia appraised their own coping skills as improved by the intervention. ${ }^{20}$ In a study where the aim was to examine the family caregivers' apprehension regarding what type of support they found important, the results revealed that the family caregivers rated support and service within the areas of information, relief, and counseling as highly important. ${ }^{21}$ This corresponds with a study where family caregivers communicated the need for better knowledge of the disease, as well as education on ways to handle cognitive and different behavioral disorders. ${ }^{22}$ In our study, the findings suggest that increased understanding gained from psychosocial intervention can help the family caregiver to better identify and describe intellectual and emotional symptoms specifically. Moreover, this might also be an explanation of the positive effect on family caregivers' burden and satisfaction ratings previously shown by Andrén and Elmståhl. ${ }^{7}$ 


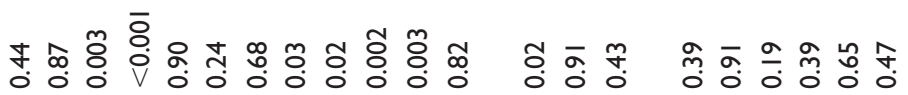

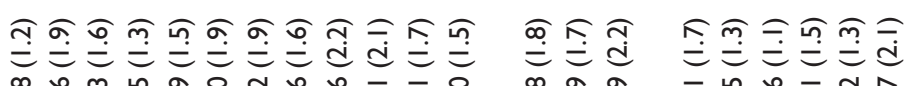

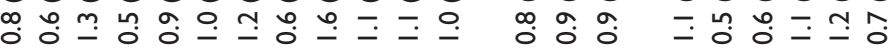

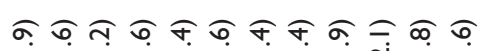

过

ช. โุด

F⿻

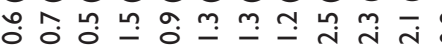

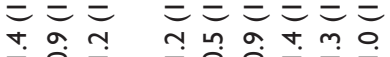

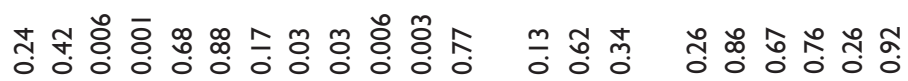

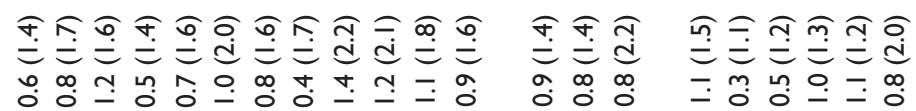

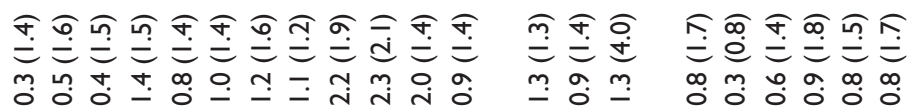

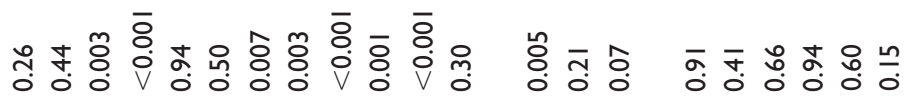

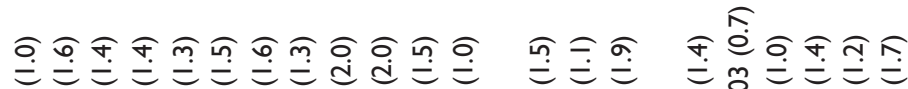

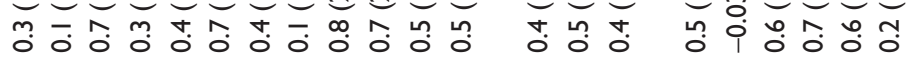

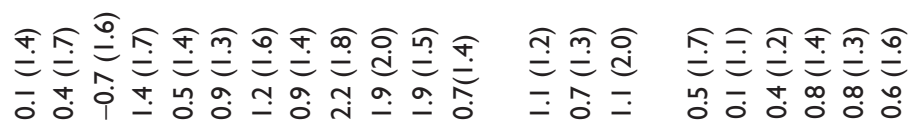

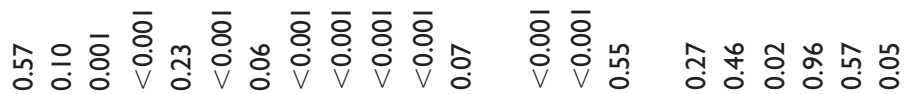

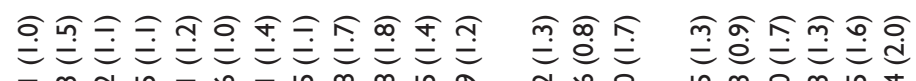

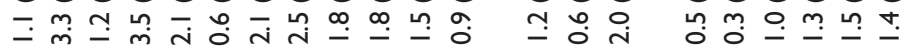

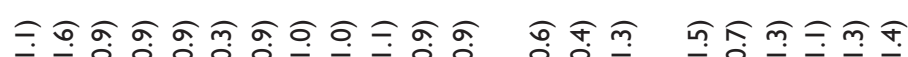

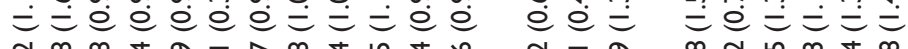

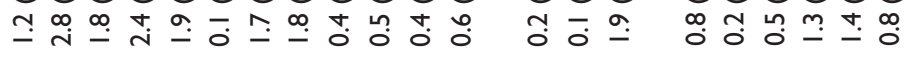

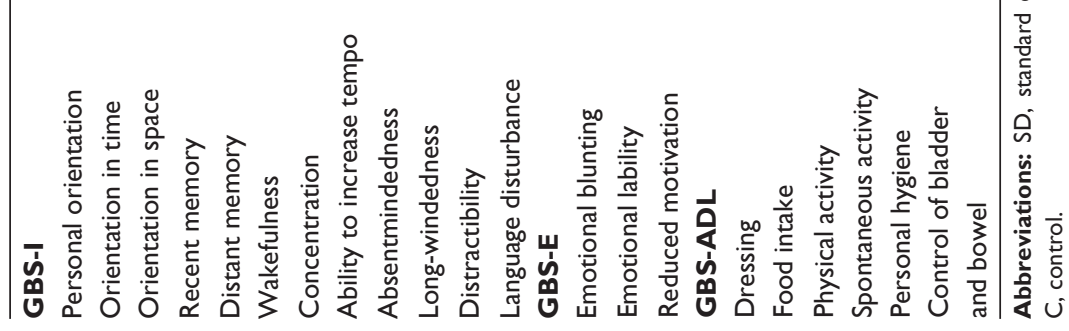



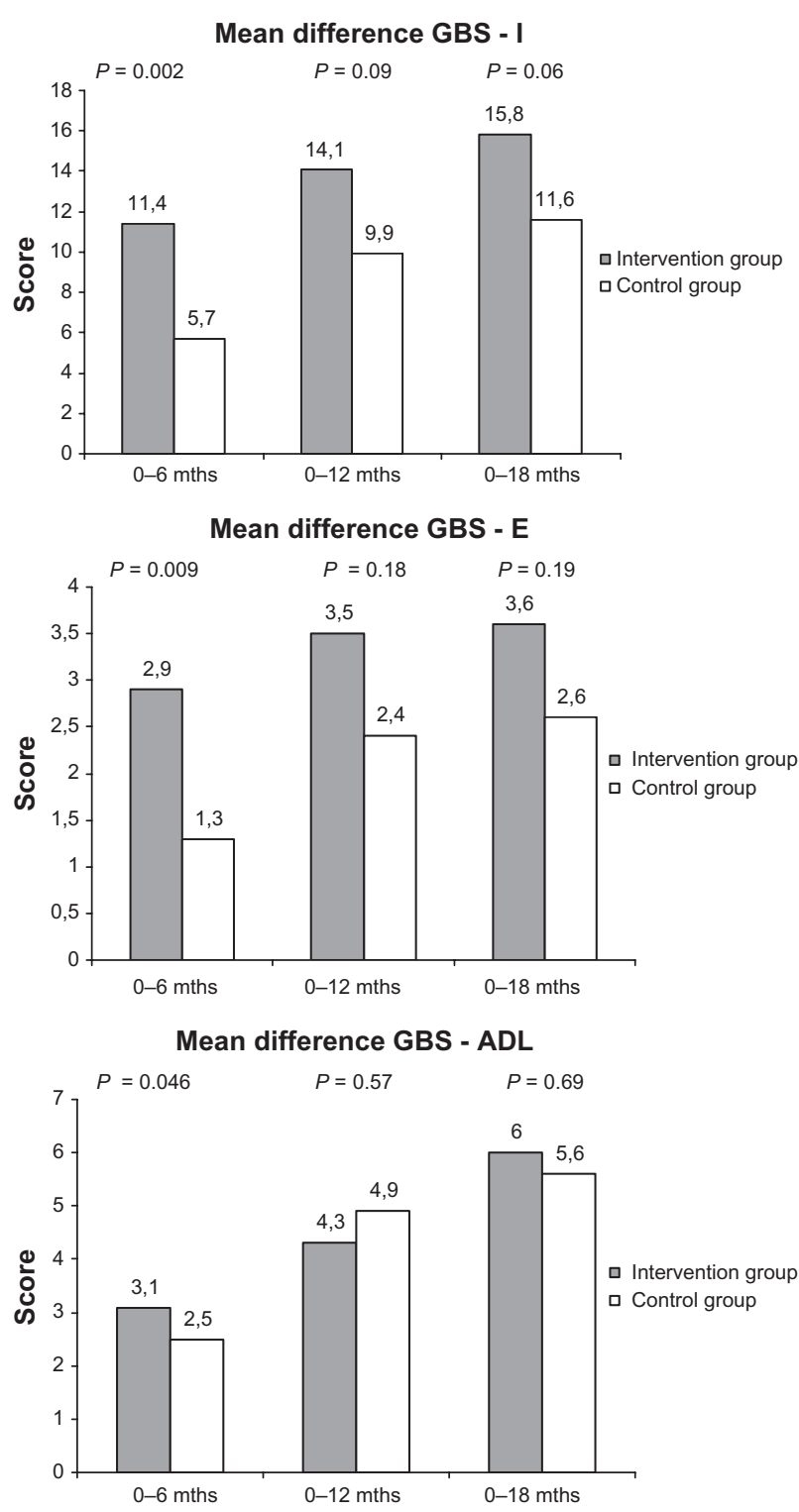

Figure I Mean differences of the ratings in the GBS-I, GBS-E, and GBS-ADL subscales by the family caregivers in the intervention group and control group, between baseline and 6, 12 and 18 months respectively for the survivors.

Abbreviations: GBS-I, Gottfries-Bråne-Steen scale-intellectual; GBS-E, GottfriesBråne-Steen scale-emotional; GBS-ADL, Gottfries-Bråne-Steen scale-activity of daily living; mths, months.

Our results indicate that it is mainly the intellectual and, to some extent, the emotional symptoms that become more evident to the family caregivers after psychosocial intervention. These symptoms are often less obvious and when they do appear are perhaps misinterpreted as general signs of old age. At baseline, assessment according to the Berger scale showed low severity of dementia in the majority of the persons with dementia in the intervention group, which correlates with the assessments of the GBS-I and GBS-E subscales. Assessment of symptoms with the Berger scale continued to reflect the ratings in the intellectual and emotional subscales of the GBS-scale in both groups during the follow-ups. The assessment of ADL functions assessed by the GBS-ADL subscale seemed to be more stable in both intervention and control groups during the studied period and this correlated with the ratings in the Katz index of ADL. Furthermore, lack of functioning in ADL is more obvious and easier to detect by the family caregiver and perhaps relates to a progression of the disease irrespective of the intervention. Both groups were rated as mildly impaired at baseline in all three subscales according to the categorization by Bråne. ${ }^{23}$ Minor differences of mean scores of subscales were noted at baseline.

Mean age and MMSE scores were almost the same among those with dementia. The baseline assessments of the GBS-scale were performed by only a few experienced RNs together with the family caregivers. In the intervention group, the same RN performed all assessments. Since assessment was performed by a small number of professionals, it is expected to have reduced the risk of measurement errors. The noted difference between both groups leveled out during the follow-up and at 18 months. The differences were small, indicating an expected progression of the disease.

At 18 months, the family caregivers were reduced to $55 \%$ because the persons with dementia had either moved to a nursing home or deceased and an inclusion criterion to participate was to care for the person living in his or her home. We can assume that the person cared for at the 18-month follow-up was less affected by dementia and this may have influenced the results. However, the result at baseline, 6, and 12 months was similar when we reduced the participants to comprise only the 18 -months survivors $(n=144)$ compared to the all participants in the study.

Our findings also revealed that kinship between the dyads or educational level among the family caregivers had no significant importance on the results in this study. This differs from previous findings showing that adult children were less accurate in their assessment than spouses. ${ }^{4}$ We found only a few significant differences in rating individual items in the subscales between spouses and adult children. One of the differences was wakefulness, a symptom that requires spending time together to be able to observe. This item was specifically noticed by spouses but seldom by adult children. Our results indicate that information, education, and discussion were effective in increasing the understanding about the occurrences of symptoms. We found no significant differences in ratings of symptoms of dementia between the participants in the intervention group who later continued with the conversation group compared to those who did not. However, we can speculate on the effect of joining a conversation 
group regarding the family caregivers' perceived burden and satisfaction presented in earlier studies, including the same participants of this study. $6,7,24$

In this study, we chose a quasi-experimental design. This design includes manipulation of the independent variable for one of the groups taking part in a psychosocial intervention. The groups were not randomly assigned. It was important to keep them unaware of each other during the relatively long follow-up period. The persons with dementia were recruited from two districts from the same municipality in southern Sweden and they were enrolled by a letter in which the intention of the study including the involvement of to the family caregiver was described. The organization and availability to public services was similar between the two districts. Also, sociodemographic characteristics were similar with respect to age and gender distribution, cohabitation, and economical resources. Furthermore, characteristics of the persons with dementia were similar regarding sociodemographics, MMSE-score as a proxy for cognitive functioning, and Katz index as a proxy for physical functioning. The participants with dementia were recruited among people already in need of help from the social services in the municipality and were identified by those providing the help. While some of the individuals had extensive need of help and support, for others it was limited to a kind of emergency call system. It was important that the persons with dementia were well-matched to enable us to study the effect of the intervention. It is possible that the family caregivers connected to persons with a greater need of help were more motivated to take part in the study, but since the groups were well-matched, any effect on the results should have been limited. Other possible confounders might be that the family caregivers in both groups were contacted by a RN by telephone every 6 months in connection with the postal questionnaire and also between the questionnaires to update changes in the care provided by the municipality and/or the family caregiver. The participants in both groups were also able to contact us for ad hoc consultation. This, together, could induce an effect on the control group similar to that in the intervention. However, this effect would merely decrease that of the intervention and reduce noted differences, especially regarding what typical symptoms could be identified, ie, distractibility, ability to increase tempo, or blunting.

The family caregivers' assessment of symptoms in the GBS-scale correlated well with the results in the Berger scale and Katz index of ADL. The results revealed that a decrease in intellectual and emotional function dominated while a decrease in ADL-function was less pronounced.
The three GBS-subscales and their individual items give a more subtle picture regarding different symptoms of dementia and help to identify and follow the progression of the disease. The GBS-scale can serve as a guide in education and information to both family caregivers and professionals. The scale was originally developed to be used by physicians, psychologists, and registered nurses after proper education. ${ }^{11}$ In this study, the GBS-scale has been shown to be a valuable tool for family caregivers in assessing different symptoms also. Undoubtedly, knowledge and understanding about the individual with dementia is crucial in assessing the symptoms. The family caregivers did not get any education prior to using the instrument. This might have aggravated the assessment.

In Sweden, social support and health care are, by law, a public responsibility and give every individual the right to apply for assistance from social services, health, and medical services. ${ }^{25}$ In spite of this, it is the family caregiver, usually a spouse or an adult child, who bears the main responsibility for the person with dementia living at home. ${ }^{26}$

The family caregivers' need of knowledge and different kinds of support has been brought to public attention and, since July 2009, it is a lawful right for family caregivers in Sweden to get information from the municipality regarding available services and support needed. The Social Service Act does not specify what kind of support the municipality shall offer, but stipulates that it must be based on individual needs. ${ }^{27}$ It is important to consider that family caregivers may have unique needs and these may change throughout the caregiving experience.

The intentions of the Social Service Act are carried out by each municipal social welfare committee and they now face the difficulty of investigating the individual needs of the family caregivers, the kind of help and support they demand, and also to investigate the effects of different interventions. The results in this study might be of interest in this process.

\section{Conclusions}

The family caregivers' knowledge and understanding about dementia disorders and their symptoms are essential in clinical settings where proxy information is an important and valued resource. In this study, our findings suggest that increased understanding gained from psychosocial intervention can help the family caregiver to better identify and describe intellectual and emotional symptoms, especially. This understanding is perhaps a plausible explanation to support earlier findings by Andrén and Elmståhl, demonstrating feelings of less burden and higher satisfaction among family caregivers who underwent this type of intervention, as well as a prolonged time to nursing placement. ${ }^{7,8}$ 
Further studies might indicate whether a caregiver's increased knowledge and understanding have an effect on the quality of life for the person with dementia and possible influences on health care costs.

\section{Description of authors' roles}

S Andrén and B Dahlrup gathered the data and analyzed the data together with E Nordell. S Andrén provided the intervention. B Dahlrup wrote the manuscript with assistance of E Nordell, who also contributed in editing the manuscript. S Elmståhl supervised the project.

\section{Acknowledgments}

This study was financed by the Medical Faculty of Lund University.

\section{Disclosure}

The authors declare no conflicts of interest in this work.

\section{References}

1. (SBU) Sbfmu, Care TSCoTAiH. SBU. Dementia. Etiology and epidemiology. A systematic review. 2008. Report No.:172E/1-3.

2. Kiyak HA, Teri L, Borson S. Physical and functional health assessment in normal aging and in Alzheimer's disease: self-reports vs family reports. Gerontologist. 1994;34(3):324-330.

3. Onor ML, Trevisiol M, Negro C, Aguglia E. Different perception of cognitive impairment, behavioral disturbances, and functional disabilities between persons with mild cognitive impairment and mild Alzheimer's disease and their caregivers. Am J Alzheimers Dis Other Demen. 2006;21(5):333-338.

4. Loewenstein DA, Argüelles S, Bravo M, Freeman RQ, Argüelles T, Acevedo A, et al. Caregivers' judgments of the functional abilities of the Alzheimer's disease patient: a comparison of proxy reports and objective measures. J Gerontol B Sci Soc Sci. 2001;56B(2):78-84.

5. Conde-Sala JL, Garre-Olmo J, Turro-Garriga O, Vilalta-Franch J, Lopez-Pousa S. Quality of life of patients with Alzheimer's disease: differential perceptions between spouse and adult child caregivers. Dement Geriatr Cogn Disord. 2010;29(2):97-108.

6. Andrén S, Elmståhl S. Family caregivers' subjective experiences of satisfaction in dementia care: aspects of burden, subjective health and sense of coherence. Scand J Caring Sci. 2005;19(2):157-168.

7. Andrén S, Elmståhl S. Psychosocial intervention for family caregivers of people with dementia reduces caregiver's burden: development and effect after 6 and 12 months. Scand J Caring Sci. 2008;22(1): 98-109.
8. Andrén S, Elmståhl S. Effective psychosocial intervention for family caregivers lengthens time elapsed before nursing home placement of individuals with dementia: a five-year follow-up study. Int Psychogeriatr. 2008;20(6):1177-1192.

9. Folstein MF, Folstein SE, McHugh PR. "Mini-mental state". A practical method for grading the cognitive state of patients for the clinician. J Psychiatr Res. 1975;12(3):189-198.

10. Diagnostic and statistical manual of mental disorders (DSM IV). Washington, DC: American Psychiatric Association;1994.

11. Gottfries CG, Brane G, Gullberg B, Steen G. A new rating scale for dementia syndromes. Arch Gerontol Geriatr. 1982;1(4):311-330.

12. Berger EY. A system for rating the severity of senility. JAm Geriatr Soc. 1980;28(5):234-236.

13. Katz S, Ford AB, Moskowitz RW, Jackson BA, Jaffe MW. Studies of illness in the aged. The index of ADL: a standardized measure of biological and psychosocial function. JAMA. 1963;21(9);185:914-919.

14. Tombaugh TN, McIntyre NJ. The mini-mental state examination: a comprehensive review. J Am Geriatr Soc. 1992;40(9):922-935.

15. Bråne $\mathrm{G}$, Gottfries $\mathrm{CG}$, Winblad $\mathrm{B}$. The Gottfries-Bråne-Steen scale: validity, reliability and application in anti-dementia drug trials. Dement Geriatr Cogn Disord. 2001;12(1):1-14.

16. Annerstedt L, Elmståhl S, Ingvad B, Samuelsson SM. Family caregiving in dementia- an analysis of the caregiver's burden and the "breakingpoint" when home care becomes inadequate. Scand J Public Health. 2000;28(1):23-31.

17. Londos E, Passant U, Brun A, Gustafson L. Clinical Lewy body dementia and the impact of vascular components. Int $J$ Geriatr Psychiatry. 2000;15(1):40-49.

18. Wallace M, Shelkey M. Reliability and validity of Katz ADL index. AJN Am J Nurs. 2008;108(4).

19. Pinquart M, Sörensen S. Helping caregivers of persons with dementia: which interventions work and how large are their effects? Int Psychogeriatr. 2006;18(4):577-595.

20. Brodaty H, Green A, Koschera A. Meta-analysis of psychosocial interventions for caregivers of people with dementia. J Am Geriatr Soc. 2003;51(5):657-664.

21. Alwin J, Oberg B, Krevers B. Support/services among family caregivers of persons with dementia - perceived importance and services received. Int J Geriatr Psychiatry. 2009;25(3):240-248.

22. Rosa E, Lussignoli G, Sabbatini F, Chiappa A, di Cesare S, Lamanna L, et al. Needs of caregivers of the patients with dementia. Arch Gerontol Geriatr. 2010;51(1):54-58.

23. Bråne G. Att bedöma demens med GBS-skalan [Assessment of dementia by using the GBS-scale]. 1 ed. Stockholm, Sweden: Natur och Kultur; 1997.

24. Andrén S, Elmståhl S. The relationship between caregiver burden, caregivers' perceived health and their sense of coherence in caring for elders with dementia. J Clin Nurs. 2008;17(6):790-799.

25. The Social service act (Socialtjänstlagen), 2001.

26. Johansson L. Anhörig- omsorg och stöd [Family member-care and support]. 1 ed. Lund: Studentlitteratur; 2007.

27. The Social service act (Socialtjänstlagen), 2001:453 Chap 5, 2009.
Clinical Interventions in Aging

\section{Publish your work in this journal}

Clinical Interventions in Aging is an international, peer-reviewed journal focusing on evidence-based reports on the value or lack thereof of treatments intended to prevent or delay the onset of maladaptive correlates of aging in human beings. This journal is indexed on PubMed Central, MedLine, the American Chemical Society's 'Chemical Abstracts Ser-

\section{Dovepress}

vice' (CAS), Scopus and the Elsevier Bibliographic databases. The manuscript management system is completely online and includes a very quick and fair peer-review system, which is all easy to use. Visit $\mathrm{http}: / /$ www.dovepress.com/testimonials.php to read real quotes from published authors. 